\title{
Complimentary Diagnostic Tools for Endometrosis in Biopsies of Mares with Clinical Subfertility
}

\author{
Daniela Rojas', Joel Cabezas², Ghyslaine Ramírez², \\ Fernando Saravia ${ }^{2}$, Lleretny Rodriguez-Alvarez² \& Fidel Ovidio Castro²
}

\begin{abstract}
Background: Endometrosis is a multifactorial disease and one of the main causes of infertility in mares, its etiology and pathogenesis are not completely understood. It is defined as peri glandular and/or stromal endometrial fibrosis with glandular alterations. Due to the few clinical symptoms, besides anamnesis and fertility data, endometrosis requires histological confirmation. The histo-morphology and immune histochemical characteristics of the endometrium vary among individuals according to the disease progression. The aim of this research was to combine histology with new immune and histochemical tools for a more precise detection of fibrotic changes of mares with endometrosis.
\end{abstract}

Materials, Methods \& Results: The endometrium of forty thoroughbred mares aged 5-18 years, that did not become pregnant during the last two breeding seasons in a Chilean commercial equine breeding center were biopsied. Samples were subjected to conventional histopathology with hematoxylin-eosin as well as to specific histological staining using specific techniques such as Alcian blue and Masson Fontana, aimed to ascertain what types of mucopolysaccharides were present in those samples. In order to have a deeper picture of the progression of the pathology, immune histochemical methods for the detection of vimentin, cytokeratin, progesterone receptor and lymphocyte marker CD3 were used. Finally in order to detect fibrillar collagen we used second harmonic generation (SHG) technique with detects fibrillar collagen without staining, due to intrinsic hyperpolarization ability of this type of collagen, which can be detected by atomic force microscopy. As a result of our research samples were categorized according to the scale of Keeney and Doig into categories I, IIa, IIb and III $(45,42,7.5$ and $5 \%$ respectively). These samples also were characterized by the methods listed earlier and a result we found specific staining in 15 samples coming from higher endometrial damage using Masson-Fontana, while acid staining indicative of acid mucopolysaccharides were detected in $97 \%$ of the samples. At immunostaining, we found cytokeratin and vimentin differentially expressed as a function of the degree of the lesions. Cytokeratin was detected in glands from healthy mares, but it was almost absent as the injury was increased. Vimentin in turn, was detected in 26 samples with different degree of intensity. In nine animals, vimentin expression was anomalous, and the marker was detected in fibrotic foci concentrically surrounding glands in biopsies of grade I and IIA only. Although progesterone receptor was detected, there was no correlation with endometrosis. Finally, the lymphocyte T specific CD3 marker was positive in 100\% of cases analyzed in which moderate lymphocytic infiltration was found by hematoxylin-eosin; however no staining could be detected in mares with more advanced endometrosis. By combining immunofluorescence with the detection of second harmonic it was possible to detect in the same sample two proteins and collagen deposition at the same time, this had not been reported earlier for endometrosis and mares.

Discussion: Our results suggest that the combination of the different methods mentioned is useful for validation and further characterization of the routine hematoxylin-eosin, and can be used as a complementary tool for the diagnosis. Of value, it was possible to combine immunofluorescence with SHG in a single sample in situ.

Keywords: biopsy, diagnosis, immunochemistry, mare endometrosis, second harmonic generation. 


\section{INTRODUCTION}

Endometrosis is a uterine fibrosis with glandular alterations $[21,25,40]$ and infiltration of immune cells [7] seriously compromising endometrial glands $[15,19,23]$ and causing infertility in mares [43]. In endometrosis, fibroblasts are converted to myofibroblast by smooth muscle actin [24,27]. Of importance to endometrosis is deposition of collagen I $[4,13]$. If myofibroblasts are not degraded, fibrosis progresses, collagen is not eliminated, resulting in endometrosis [29]. Biopsy score is the gold standard for evaluating endometrial tissue health $[8,18,20]$. There are 4 categories of endometrosis: being I the normal and III the severe damaged endometrium. IIa and $\mathrm{b}$ stand for intermediate damages [20,38]. Immune detection of steroid receptors, [23] cytokeratin, vimentin $[1,10,26]$ and fibrillar collagen (FC) may be of help in diagnostic. FC is able of hyperpolarization leading to second harmonic generation properties (SGH) [11] a laser-dependent process based on nonlinear excitation measurable with multiphoton microscopy [41]. This provides a great tool for imaging tissue structure with submicron resolution of thick specimens $[6,41,44]$. It has been used to document collagen fibers in different fibrosis and connective tissue disorders [4]. The aim of this research was to combine histology with new immune and histochemical tools for a more precise detection of fibrotic changes of mares with endometrosis. Of particular interest was the use of vimentin and estrogen receptor as markers of progression of the disease and the use of SGH properties of fibrillar collagen to a more robust classification of endometrosis in barred mares by analyzing a single biopsy sample.

\section{MATERIALS AND METHODS}

\section{Animals and biopsies}

Forty Thoroughbred English blood race mares, 8 to 15 years old, in different stages of the estrous cycle, belonging to a Chilean commercial equine breeding center in the city of Los Angeles, VIII region Bío-Bío, Chile were biopsied. All mares had a record of at least two years in a raw of nonpregnancy in spite of scheduled mating with proven stallions. Endometrial biopsy samples were collected from all 40 mares. A punch of approximately $1 \mathrm{~cm}$ x $0.5 \mathrm{~cm}$, was taken from the uterus of mares, using biopsy forceps from binding site between the body and the right horn. All mares were examined by vaginoscopy and ultrasonography to detect the state of the estrus cycle and the absence of concomitant cysts or infections.

\section{Histology}

The biopsies were fixed in $10 \%$ buffered formalin, embedded in paraffin (FFPE), sectioned at $4 \mu \mathrm{m}$ and stained with hematoxylin-eosin (H-E) and analyzed by light microscopy. The pathological alterations of the endometrium were graded according to the system of Kenney and Doig [20,35].

\section{Histochemistry}

Alcian blue staining at $\mathrm{pH} 2.5$ was used for the detection of acid mucopolysaccharides. Masson's trichrome stain (MTS) was used to characterize and identify collagen deposits in the glands and the stromal tissue. (MTS was performed according to Army Forces Institute Pathology, AFIP).

\section{Immunohistochemistry}

Tissue sections were mounted on Epic plus slides ${ }^{1}$. The paraffin wax sections were rehydrated and endogenous peroxidase activity was inhibited by $3 \% \mathrm{H}_{2} \mathrm{O}_{2}$ in methanol for $30 \mathrm{~min}$. The antibodies used were diluted in Diamond antibody diluent ${ }^{2}$. Antibodies and dilutions used were: vimentin, monoclonal mouse clone V9 (cat No. M0725) 1:200, cytokeratin monoclonal mouse clones AE1/AE3 (cat No. M3515) 1:200, CD3; polyclonal rabbit (cat No. A04523), progesterone receptor; monoclonal rabbit clone Y85 (cat No. 323R$17)^{2}$ ready to use, estrogen receptor; monoclonal rabbit clone SP1(cat No. 249R-18) ${ }^{2}$ ready to use. When appropriate, pretreatments for antigen retrieval were applied with EDTA pH 8.0 at $95^{\circ}-100^{\circ} \mathrm{C}$ for $10 \mathrm{~min}$. The antibodies were incubated at $4^{\circ} \mathrm{C}$ overnight. The Labelled Streptavidin-Biotin Horseradish Peroxidase System 2, (LSAB2 System, HRP) ${ }^{3}$ was used for imunohistochemestry. After each step, sections were rinsed thoroughly with PBS. The slides were developed in diaminobenzinetetrahydrochloride (DAB ImmPact) ${ }^{4}$. The sections were counterstained with hematoxylin, then cleared and mounted.

\section{Immunofluorescence}

Second Harmonic Generation was used to visualize the collagen fibers, for this the samples were analyzed with a pulsed laser in a confocal microscope 
LSM7005. Images were acquired and treated with the built-in processing software. Briefly, the $4 \mu \mathrm{m}$ samples from FFPE were dewaxed, hydrated and incubated in $1 \%$ PBS-Tween-202 for 20 min at room temperature. Then, protein blocking was performed with $3 \%$ normal horse serum, by incubating overnight at $4{ }^{\circ} \mathrm{C}$, with the following mixtures of antibodies: anti-Cytokeratin ${ }^{3}$ (1:200 rabbit) and anti-Vimentin ${ }^{3}$ (1:200 mouse). On the second day samples were washed thoroughly and then incubated with secondary Alexa Fluor 4881 anti rabbit (1:200) and Alexa Fluor 5941 anti mouse (1:200) in the same reaction, for one $\mathrm{h}$ at room temperature in the dark. Then the samples were washed in PBS, and mounted with prolong gold antifade reagent with DAPI $^{6}$ aqueous mounting medium. Immunofluorescence with the antibodies listed above was performed on the same samples at the same time.

\section{Statistical analysis}

All associations analyses were performed with Spearman correlation test using Infostat ${ }^{7}$ Correlations analyzed were: H\&E, Masson Fontana and vimentin expression and $\mathrm{P} 4 \mathrm{R}$ expression and degree of endometrosis.

\section{RESULTS}

\section{Histology}

Processed samples from FFPE were categorized according to literature [20,35]. Representative microphotographs (H\&E) are shown in Figure 1 a, f, $\mathrm{k}$ and $\mathrm{p}$. The distribution of the grading was: 45,42 , 7.5 and $5 \%$ for grades I, IIA, IIB and III respectively (Table 1).

\section{Histochemistry}

In the Masson-Fontana trichrome assay (Figure $1 \mathrm{~b}, \mathrm{~g}, 1$ and q) positive staining was detected in 15 samples, which had higher endometrial damage. In the samples stained with Alcian Blue, acid mucopolysaccharides were detected in $97 \%$ of the cases analyzed, located mainly in luminal epithelium and superficial glands. In eight samples (20\%) acid mucin was detected in deep areas what is considered abnormal (Figure $1 \mathrm{c}, \mathrm{h}, \mathrm{m}$ and $\mathrm{r}$ ).

\section{Immunohistochemistry}

Vimentin: The expression of vimentin was detected in 26 samples with different degree of intensity.
In nine animals, vimentin expression was anomalous, and the marker was detected in fibrotic foci concentrically surrounding glands in biopsies of grade I and IIA only (Figure $1 \mathrm{~d}, \mathrm{i}, \mathrm{n}$ and $\mathrm{s}$ ).

Cytokeratin: this epithelial marker was expressed differently according to the grade of endometrosis, being stronger in glands from healthy mares, and almost absent as the injury was increased (Figure $1 \mathrm{e}, \mathrm{j}, \mathrm{o}$ and $\mathrm{t}$ ).

Progesterone receptor (P4r): the expression of the P4r marker, was found with greater intensity and frequency in the nucleus of glands and in some stromal cells of the endometrium of mares in luteal phase compared to those from the follicular phase (Figure 2). No correlation with endometrosis was detected.

CD3: The lymphocyte T specific CD3 marker was positive in $100 \%$ of cases analyzed in which moderate lymphocytic infiltration was found by hematoxylin-eosin. This infiltrate however was not found in mares with advanced endometrosis (Figure 3).

\section{Inmunofluorescence and $\mathrm{SHG}$}

By combining immunofluorescence with the detection of second harmonic it was possible to detect in the same sample two proteins and collagen deposition at the same time (Figure 4).

\section{Statistical analysis}

We studied if there were associations between the three tests used for the detection of endometrial fibrosis using Spearman correlation test. No correlation was found between vimentin expression and the degree of endometrosis according to the Keneey and Doig's scale. Vimentin expression was detected also in biopsies with absent or mild fibrosis as detected by the hematoxylin and eosin test. There was a correlation between the Masson Fontana stain and H\&E scoring $(P=0.02)$.

Vimentin expression and detection did not correlate with the degree of endometrosis, since it was detected mainly in biopsies from grade I and IIa individuals. Expression of progesterone receptor did not have a statistically significant correlation in relation to the endometrial grade in any area of the sample (glandular epithelium, luminal epithelium and stroma). Also expression of P4R, did not correlate with the expression of vimentin, age of the mares or number of fowling. 
D. Rojas, J. Cabezas, G. Ramírez, et al. 2020. Complimentary Diagnostic Tools for Endometrosis in Biopsies of Mares with Clinical Subfertility.

Acta Scientiae Veterinariae. 48: 1717.

Table 1. Grading of endometrial biopsies* and its relation with age and number of pregnancies of mares and the respective histological techniques used.

\begin{tabular}{ccccccccc} 
Category & $\begin{array}{c}\mathrm{N}^{\circ} \\
\text { mares }\end{array}$ & Age (years) & $\mathrm{N}^{\circ}$ pregnancies & $\begin{array}{c}\text { Degenerative } \\
\text { changes }\end{array}$ & Vimentin & Cytokeratin & $\begin{array}{c}\text { Masson } \\
\text { fontana }\end{array}$ & Alcian blue \\
\hline I & 18 & $11.1 \pm 3.9$ & $4.6 \pm 2.7$ & Absent & - & +++ & + Absent \\
IIA & 18 & $11 \pm 3.7$ & $4.4 \pm 2.7$ & Medium & ++- & +++ & + & Absent \\
IIB & 3 & $10.3 \pm 3.9$ & $4.1 \pm 2.7$ & Moderate & ++++ & ++ & ++ & Present \\
III & 1 & 14 & 0 & Severe & +++ & $+/-$ & +++ & Present \\
\hline
\end{tabular}

*According to Keneey and Doig [20].

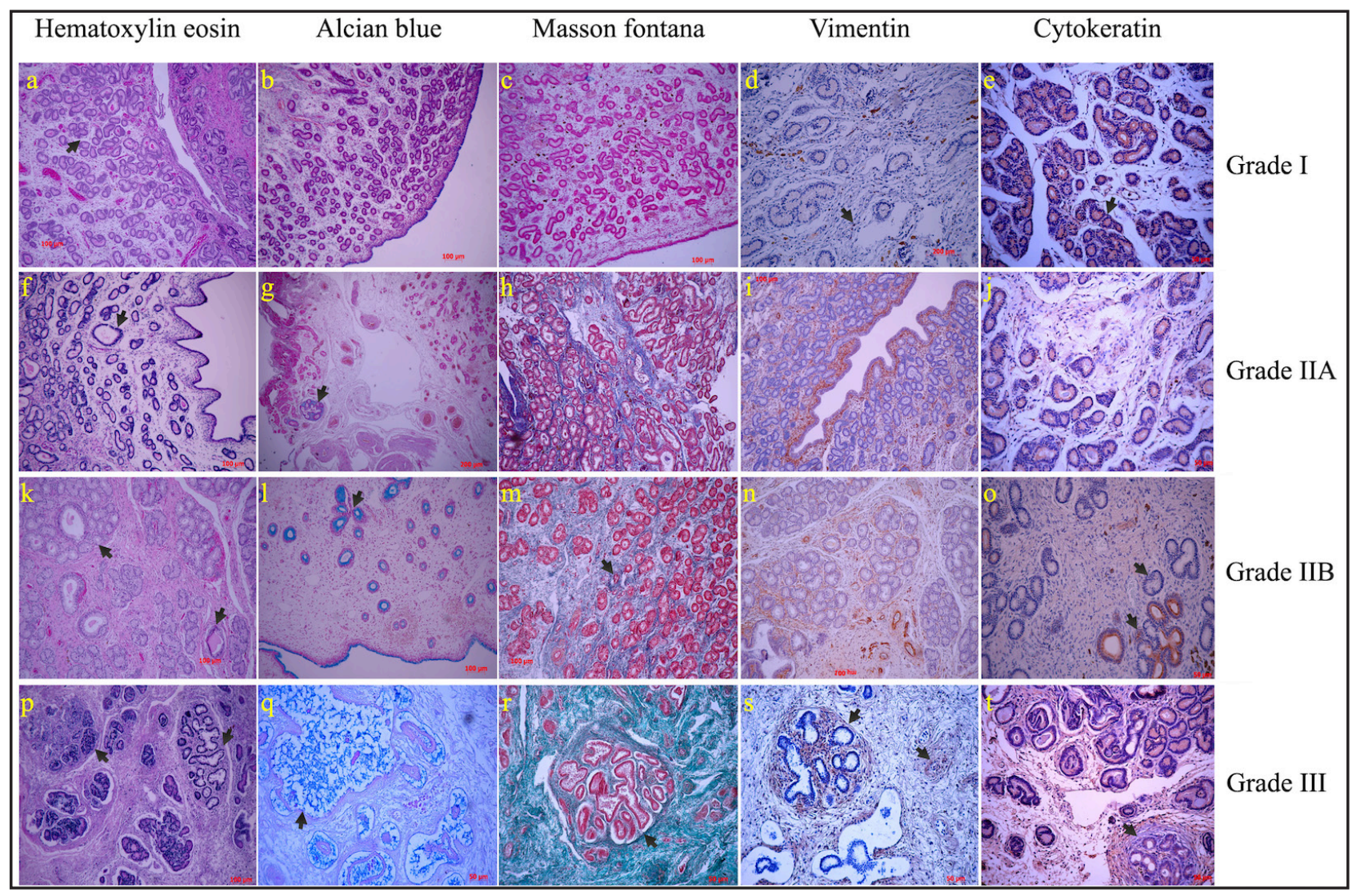

Figure 1. Representative histological sections of biopsies of mares, processed with different histological techniques. H\&E; well-distributed and organized glands in grade I (a; arrow) and IIA (f), a slight formation of cysts in the glands is pointed by an arrow in (f). In IIB formation of glandular nests encysting of the glands (arrows in k) with an evident loss of the endometrial architecture. Total loss of the normal architecture of the endometrium can be appreciated in the degree III (p), with evident formation of glandular nests and glandular cysts. In the Alcian Blue stain, a normal architecture can be seen in b (grade I), without the presence of mucopolysaccharides, in the degree IIA (g) the architecture is maintained but mucopolysaccharide deposit is present (arrow). In grade IIB, such deposits are more abundant (1) and are spread all over the sample in grade III (q). In the Masson Fontana stain $(\mathrm{c}, \mathrm{h}, \mathrm{m}, \mathrm{r})$, the accumulation of collagen, is detected in the samples with grade IIA (h), and its increased in the more damaged endometria of $\mathrm{m}$ and $\mathrm{r}$. Immunohistochemistry for vimentin $(\mathrm{d}, \mathrm{i}, \mathrm{n}, \mathrm{s})$, and cytokeratin $(\mathrm{e}, \mathrm{j}, \mathrm{o}, \mathrm{t})$. A gradual accumulation of collagen from grade IIA to grade III is appreciated together with the increase in the degree of endometrosis, while cytokeratin expression decreases (arrows). [Magnification of 100x for all images]. 
D. Rojas, J. Cabezas, G. Ramírez, et al. 2020. Complimentary Diagnostic Tools for Endometrosis in Biopsies of Mares with Clinical Subfertility.

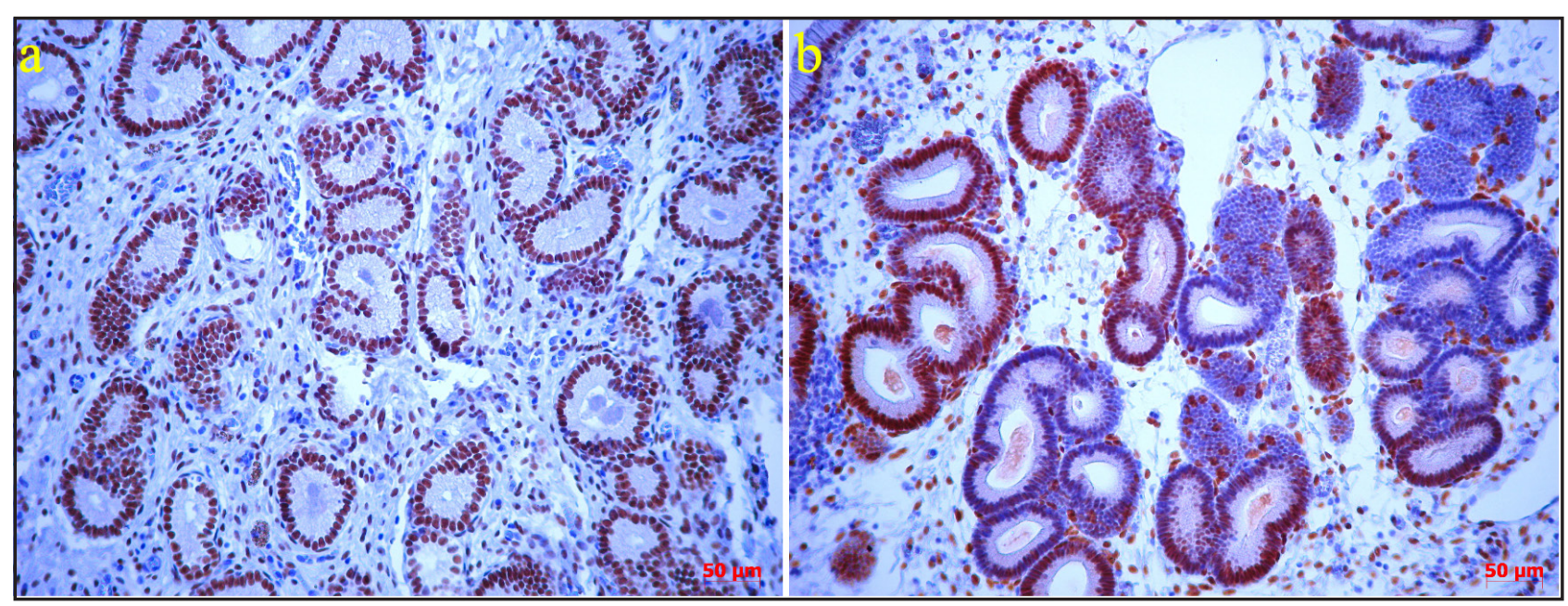

Figure 2. Immunostaining of progesterone receptor expression. a) Intense immunostaining in nucleus of glands, as well in stroma of grade I mare sample. b) Immunostaining is observed in some glands of grade III mare samples. [Magnification of 200x for all images].

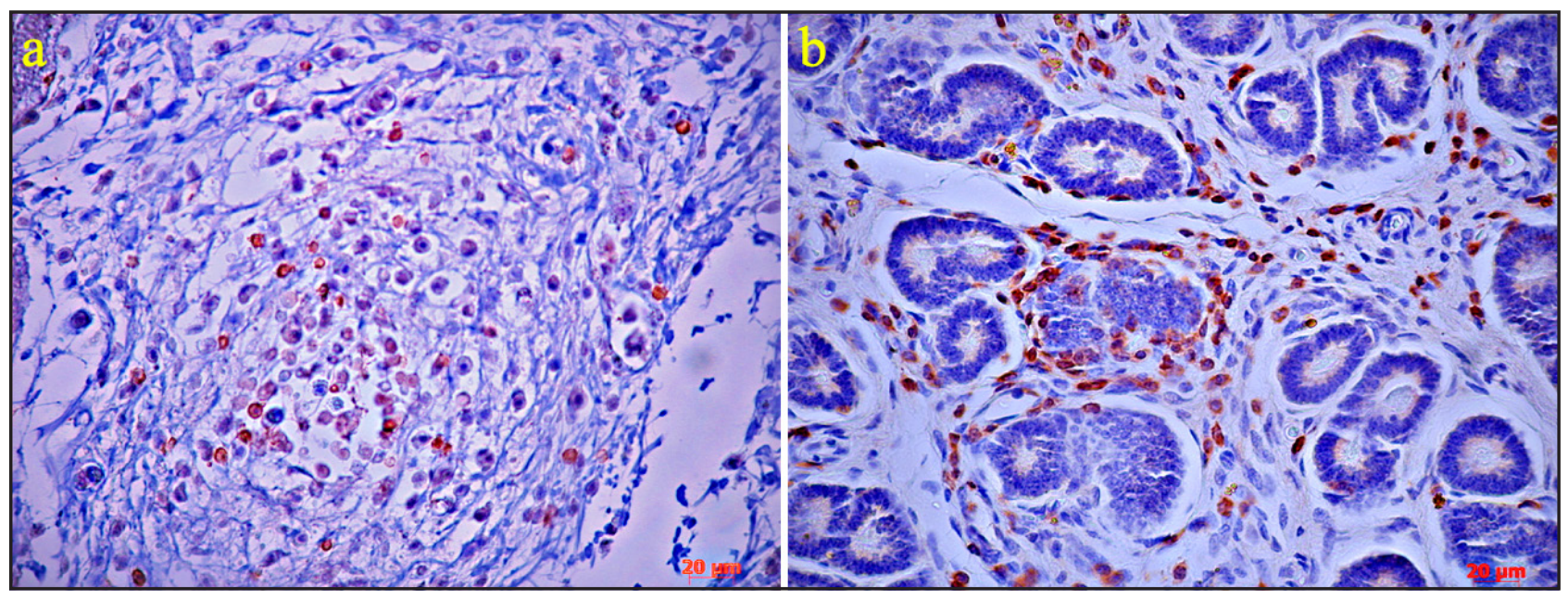

Figure 3. Immunohistochemical staining for detection of CD3 antigen. Distribution of T lymphocyte cells in biopsies with different grade pathological (a IIb ,b I). [Magnification of 400x for all images].

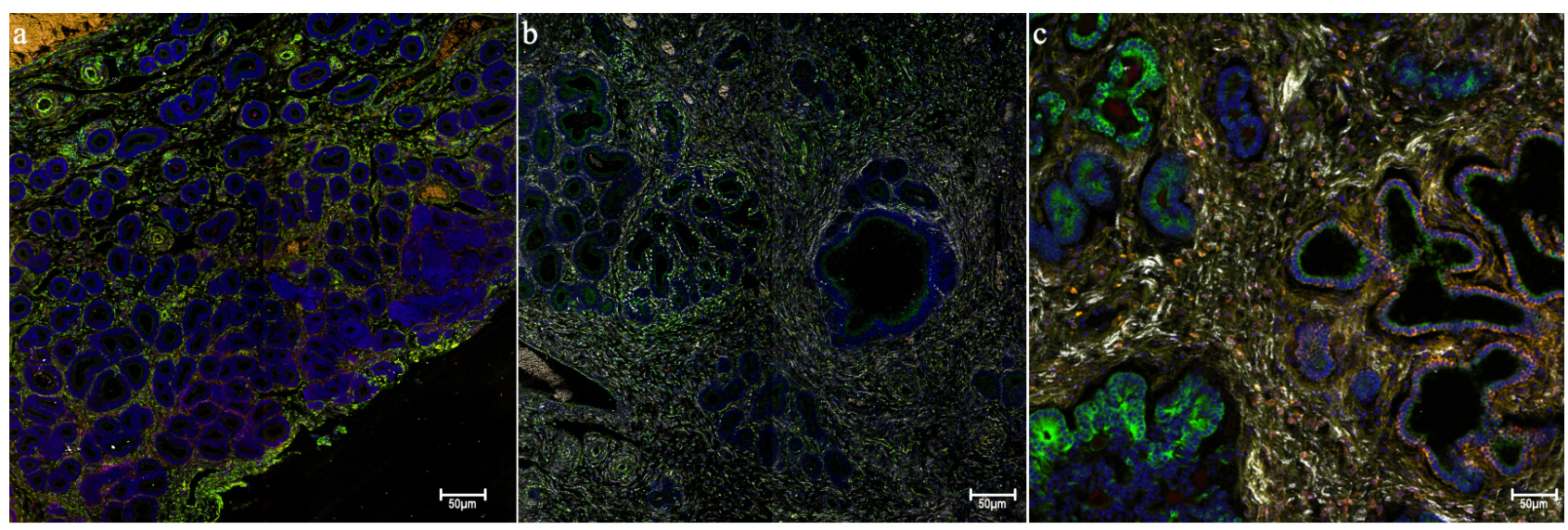

Figure 4. Spectral microscopy images of second harmonic generation; a) Mare biopsy grade I, b) mare biopsy Grade IIB, c) and mare biopsy grade III. Green: cytokeratin; red: vimentin; white: excited collagen fibers with polarized light and blue: nuclear staining with DAPI. [Magnification of 250x for all images]. 


\section{DISCUSSION}

As of today, histopathological analysis is the most specific test to detect endometrosis and other disorders responsible for huge losses in reproduction and equine breeding. Subclinical endometrosis represents a great diagnostic challenge and is one of the most important reasons for subfertility in mares. This disease, together with endometritis of infectious origin and the associated inflammatory and degenerative changes, are the most important triggering factors that generate a marked impact on the fertility of mares [1,5,30,31].

In this study, endometrial biopsies were obtained from 40 Thoroughbred English blood race mares, with a history of subfertility of at least two years and which did not become pregnant in the previous season. In addition to traditional laboratory diagnosis based on routine histology, biopsies were also subjected to immunohistochemical tests and special staining as a possible diagnostic complement of subfertility. The histopathological evaluation by hematoxylin and eosin staining is considered the reference standard for the diagnosis of endometritis, degenerative changes and other endometrial alterations [30].

In the routine $\mathrm{H} \& \mathrm{E}$ staining the distribution of the grading was: $45,42,7.5$ and $5 \%$ respectively for grades I, IIa, IIb and III respectively. This denotes that most of the sampled animals did not present severe histological alterations. Others reported in previous studies $[33,43]$ a significant association between the degree of endometrosis and the age of the mare. This was not the case in the current study.

Acid mucopolysaccharides were detected in $97 \%$ of the cases analyzed, located mainly in luminal epithelium and superficial glands, which according to Walter [39] is considered normal. However, this study corroborates the findings of Hoffmann [16] since accumulations of acid mucin were also associated with deep glandular nests with the presence of glandular dilation. In contrast to reported in the literature [16] in the present study this phenomenon could not be related to fibrosis and endometrosis.

In women, the presence of acid mucin is indicative of adenocarcinomatosis and other severe pathologies. The analysis of acidic mucopolysaccharides is still very scarce in the equine endometrium, but it is logical to think that it could affect the uterine microenvironment and with that a future pregnancy [7]. Therefore future investigations are needed to elucidate their true implications in fertility. Alterations in the $\mathrm{pH}$ must receive an adequate treatment, and as a diagnostic method Alcian blue pH 2.5 staining could be implemented in practice in conjunction with hematoxylin and eosin staining.

Masson-Fontana trichrome staining allowed for the detection of fibrosis in the samples tested, this is in agreement with others [2] who detected an increase in fibrosis in stromal tissue using the same technique. In our case, this increase can be attributed to the specificity of Masson's stain to collagen, which can give false positives in the detection of fibrosis. From the results discussed it is proposed that the use of said staining in endometrial biopsies is not of significant benefit, being the staining of hematoxylin and eosin more accurate in the diagnosis of incidence and severity of endometrial fibrosis.

While widely used in human clinic, immunohistochemistry is not a common tool in the diagnostic of endometrial diseases in horses. Here we detected that the intermediate filament marker vimentin correlated with hematoxylin and eosin staining in the samples of animals with endometrosis (grades IIb and III), thus it can be of value for fibrosis detection; however expression was found also in samples graded as I and IIA. The expression of vimentin is indicative of the presence of fibroblasts, which may or may not be producing collagen, so finding a marked presence of fibroblasts in a histologically healthy endometrium could mean a potential early marker of fibrosis, which would be a tool for much importance in the equine industry. In recent years vimentin has gained much importance as a marker of the "epithelial-mesenchymal transition" (EMT), which is associated with a series of tumorigenic events [12,22,34].

As for the receptors to steroidal hormones, their absence or decrease can cause alterations in the function of the endometrial glands. In human gynecology these disturbances are a proven cause of infertility $[24,35]$. In the present study it was not possible to establish correlations between the expression of the progesterone receptor and the endometrial pathologies, nor with the age of the mares or number of previous fowling. One possible reason is that in all, only five animals had severe endometrosis (grades IIb and III). Here we did not analyze the expression of the estrogen receptor. Our results do not coincide with what was reported by Hoffmann et al. [14] who showed a con- 
siderable decrease in the expression of estrogen and progesterone receptors in endometrium affected by fibrosis. Again the limited amount of severe damaged samples might impinge upon the comparison.

On the other hand, the association between lymphocytic infiltrations and the presence of fibrosis has been described by different authors $[8,9,20]$. We used immunohistochemistry for the labeling of lymphocytes, by the cytoplasmic protein CD3 in the endometrial biopsies, this is not commonly found in literature dealing with mare endometrosis. These findings are indicative of chronic inflammation. There was a complete coincidence between the finding of CD3+ samples with detection of lymphocyte infiltration in the same biopsies as judged by H\&E staining. The latter technique is much more available and affordable, but definitively more subjective than that of an immunomarker. We suggest that CD3 staining might be used as a confirmatory method.

Vimentin staining could be used as a predictive marker or as a predisposition of the mare to develop a fibrosis, since its presence was detected in healthier endometria, but not in endometria with more intense (grade IIb) or severe (grade III) endometrosis. Conversely, cytokeratin can be of help for the demonstration of functionality of the glands.

In an effort to make the best use of a biopsy sample, we set out to obtain the largest amount of information in a single sample using histological tools. Is known that the amount of collagen deposited of fibroblast contribute to the formation of a permanent fibrotic scar [42], therefore a technique that allows to evaluate collagen deposits, and that can also be combined with other techniques to identify different associated markers, is necessary.
The collagen molecules organize naturally in fibers on the scale of the wavelength of light and lack inversion symmetry, thus allowing for the process of second harmonic generation (SHG) when exposed to incident light [32].

\section{CONCLUSIONS}

We combined immunohistochemistry staining for vimentin and cytokeratin with second harmonic generation for detection of collagen and with nuclear staining. The overall result allowed for a more detailed detection of collagen fibers and said proteins as a function of the degree of endometrosis. To our knowledge this had not been reported previously for endometrosis of mares.

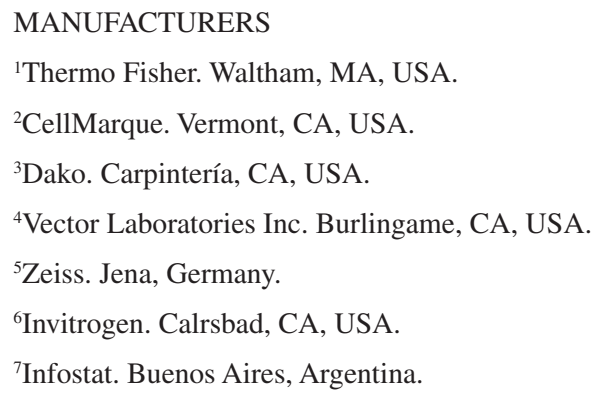

Funding. Fondecyt Regular 1150757 to F.O.C., Ministry of Education, Government of Chile

Acknowledgments. Authors thank Claudio Omon and horse staff at Haras Don Alberto, Los Angeles, VIII Region, Chile.

Ethical approval. All the procedures of this study were carried out under the approval of the Bioethics Committee of the University of Concepción, document number: CBE-13-15.

Declaration of interest. The authors declare no conflict of interest. The authors alone are responsible for the content and writing of paper.

\section{REFERENCES}

1 Aupperle H., Schoon D. \& Schoon H.A. 2004. Physiological and pathological expression of intermediate filaments in the equine endometrium. Research in Veterinary Science. 76(3): 249-255.

2 Blanchard T.L., García M.C., Kintner L.D. \& Kenney R.M. 1987. Investigation of the representativeness of a single endometrial sample and the use of trichrome staining to aid in the detection of endometrial fibrosis in the mare. Theriogenology. 28(4): 445-450.

3 Carlson M.A. \& Longaker M.T. 2004. The fibroblast-populated collagen matrix as a model of wound healing: a review of the evidence. Wound Repair and Regeneration. 12(2): 134-147.

4 Chen X., Nadiarynkh O., Plotnikov S. \& Campagnola P.J. 2012. Second harmonic generation microscopy for quantitative analysis of collagen fibrillar structure. Nature Protocols. 7(4): 654-669. 
5 Christoffersen M., Woodward E.M., Bojesen A.M., Petersen M.R., Squires E.L., Lehn-Jensen H. \& Troedsson M.H. 2012. Effect of immunomodulatory therapy on the endometrial inflammatory response to induced infectious endometritis in susceptible mares. Theriogenology. 78(1): 991-1004.

6 Denk W., Strickler J.H. \& Webb W.W. 1990. Two-photon laser scanning fluorescence microscopy. Science. 248(4951): 73-76.

7 Doig P. \& Waelchli R. 1993. Endometrial biopsy. In: McKinnon A.O. \& Voss J.L. (Eds). Equine Reproduction. Philadelphia: Lea \& Febiger, pp.225-233.

8 Ferreira-Dias G., Nequin L.G. \& King S.S. 1999. Morphologic comparisons among categories I, II, and III equine endometrium using light and transmission electron microscopy. American Journal of Veterinary Research. 60(1): 49-55.

9 Flores J.M., Rodríguez A., Sánchez J., Gómez-Cuétara C. \& Ramiro F. 1995. Endometrosis in mares: incidence of histopathological alterations. Reproduction in Domestic Animals. 30(2): 61-65.

10 Franke W.W., Schmid E., Winter S., Osborn M. \& Weber K. 1979. Widespread occurrence of intermediate-sized filaments of the vimentin-type in cultured cells from diverse vertebrates. Experimental Cell Research. 123(1): 25-46.

11 Freund I., Deutsch M. \& Sprecher A. 1986. Connective tissue polarity. Optical second-harmonic microscopy, crossedbeam summation, and small-angle scattering in rat-tail tendon. Biophysical Journal. 50(4): 693-712.

12 Gilles C., Polette M., Zahm J.M., Tournier J.M., Volders L., Foidart J.M. \& Birembaut P. 1999. Vimentin contributes to human mammary epithelial cell migration. Journal of Cell Science. 112(24): 4615-4625.

13 Greiling D. \& Clark R.A. 1997. Fibronectin provides a conduit for fibroblast transmigration from collagenous stroma into fibrin clot provisional matrix. Journal of Cell Science. 110(7): 861-870.

14 Hoffmann C., Bazer F.W., Klug J., Aupperle H., Ellenberger C. \& Schoon H.A. 2009. Immunohistochemical and histochemical identification of proteins and carbohydrates in the equine endometrium: Expression patterns for mares suffering from endometrosis. Theriogenology. 71(2): 264-274.

15 Hoffmann C., Ellenberger C., Mattos R.C., Aupperle H., Dhein S., Stief B. \& Schoon H.A. 2009. The equine endometrosis: New insights into the pathogenesis. Animal Reproduction Science. 111(24): 261-278.

16 Jielsen J.M. 2005. Endometritis in the mare: a diagnostic study comparing cultures from swab and biopsy. Theriogenology. 64(3): 510-518.

17 Kenney R.M. 1978. Cyclic and pathologic changes of the mare endometrium as detected by biopsy, with a note on early embryonic death. Journal of American Veterinary Medicine Association. 172(3): 241-262.

18 Kenney R.M. 1992. The etiology, diagnosis and classification of chronic degenerative endometritis. Equine Veterinary Journal. 25(3): 186-192.

19 Kenney R.M., Bergman R.V., Cooper W.L. \& Morse G.W. 1975. Minimal contamination techniques for breeding mares: techniques and preliminary findings. Proceedings of the American Association of Equine Practitioners. 21: 327-336.

20 Kenney R.M. \& Doig P.A. 1986. Equine endometrial biopsy. In: Morrow D.A. (Ed). Current Therapy in Theriogenology. Philadelphia: Saunders W.B, pp.723-729.

21 Kenney R. \& Ganjan V. 1975. Selected pathological changes of the mare uterus and ovary. Journal of Reproduction and Fertility. 23(Suppl): 335-339.

22 Kidd M.E., Shumaker D.K. \& Ridge K.M. 2014. The role of vimentin intermediate filaments in the progression of lung cancer. American Journal of Respiratory Cell and Molecular Biology. 50(1): 1-6.

23 Lehmann J., Ellenberger C., Hoffmann C., Bazer F.W., Klug J., Allen W.R., Sieme H. \& Schoon H.A. 2011. Morpho-functional studies regarding the fertility prognosis of mares suffering from equine endometrosis. Theriogenology. 76(7): 1326-1336

24 Lorena D., Uchio K., Costa A.M. \& Desmouliere A. 2002. Normal scarring: importance of myofibroblasts. Wound Repair and Regeneration. 10(2): 86-92.

25 Mansour G.D., Henry M. \& Ferreira A.M. 2003. Immunohistochemical study of equine endometrial extracellular matrix during the oestrous cycle. Journal of Comparative Pathology. 129(4): 316-319.

26 Moll R., Franke W.W., Schiller D.L., Geiger B. \& Krepler R. 1982. The catalog of human cytokeratins: patterns of expression in normal epithelia, tumors and cultured cells. Cell. 31(1): 11-24.

27 Mutsaers S.E., Bishop J.E., McGrouther G. \& Laurent G.J. 1997. Mechanisms of tissue repair: from wound healing to fibrosis. International Journal of Biochemistry and Cell Biology. 29(1): 5-17. 
29 Oddsdóttir C., Riley S.C., Leask R., Shaw D.J., Aurich C., Palm F., Fowden A.L., Ricketts S.W. \& Watson E.D. 2011. Dynamics of activities of matrix metalloproteinases-9 and -2, and the tissue inhibitors of MMPs in fetal fluid compartments during gestation and at parturition in the mare. Theriogenology. 75(6): 1130-1138.

30 OverbeckW., JägerK., Schoon H.A. \& Witte T.S. 2013. Comparison of cytological and histological examinations in different locations of the equine uterus-an in vitro study. Theriogenology. 79(9): 1262-1268.

31 Overbeck W., Witte T.S. \& Heuwieser W. 2011. Comparison of three diagnostic methods to identify subclinical endometritis in mares. Theriogenology. 75(7): 1311-1318.

32 Rao R.A.R., Metha M.R., Leithem S. \& Toussani K.C. 2009. Quantitative analysis of forward and backward second harmonic generation microscopy. Optical. Letters. 34(24): 3779-3381.

33 Ricketts S.W. \& Alonso S. 1991. The effect of age and parity on the development of equine chronic endometrial disease. Equine Veterinary Journal. 23(3): 189-192.

34 Satelli A. \& Li S. 2011. Vimentin in cancer and its potential as a molecular target for cancer therapy. Cellular and Molecular Life Science. 68(18): 3033-3046.

35 Schoon H.A., Schoon D. \& Klug E. 1992. Uterusbiopsien als Hilfsmittel fur Diagnose und Prognose von Fertilitatsstorungen der Stute. Pferdeheilkunde. 8(6): 355-362.

36 Tilbury K., Hocker J., Wen B.L., Sandbo N., Singh V. \& Campagnola P.J. 2014. Second harmonic generation microscopy analysis of extracellular matrix changes in human idiopathic pulmonary fibrosis. Journal of Biomed Optics. 19(8): 86014.

37 Turani H., Lurie B., Chaimoff C. \& Kessler E. 1986. The diagnostic significance of sulfated acid mucin content in gastric intestinal metaplasia with early gastric cancer. American Journal of Gastroenterology. 81(5): 343-345.

38 Waelchli R. 1990. Endometrial biopsy in mares under non uniform breeding management conditions: Prognostic value and relationship with age. Canadian Veterinary Journal. 31(5): 379-384.

39 Walter I., Handler J., Reifinger M. \& Aurich C. 2001. Association of endometrosis in horses with differentiation of periglandular myofibroblasts and changes of extracellular matrix proteins. Reproduction. 121(4): 581-586.

40 Watson E.D. \& Thomson S.R.M. 1996. Lymphocyte subsets in the endometrium of genitally normal mares and mares susceptible to endometritis. Equine Veterinary Journal. 28(2): 106-111.

41 Williams R.M., Zipfel W.R. \& Webb W.W. 2001. Multiphoton microscopy in biological research. Current Opinion in Chemical Biology. 5(1): 603-608.

42 Wynn T.A. 2007. Common and unique mechanisms regulate fibrosis in various fibroproliferative diseases. Journal of Clinical Investigation. 117(3): 524-529.

43 Zent W.W. \& Troedsson M.H. 1998. Post breeding uterine fluid accumulation in a normal population of thoroughbred mares: A field study. Proceedings of the American Association of Equine Practicioners. 44: 64-65.

44 Zipfel W.R., Williams R.M. \& Webb W.W. 2003. Nonlinear magic: multiphoton microscopy in the biosciences. Nature Biotechnology. (21): 1369-1377. 